\title{
Intra-articular hyaluronan injections in the treatment of osteoarthritis of the knee: a randomised, double blind, placebo controlled multicentre trial
}

\author{
L Stefan Lohmander, Nils Dalén, Gunnar Englund, Martti Hämäläinen, Erik Martin \\ Jensen, Kerstin Karlsson, Magnus Odensten, Leif Ryd, Ingemar Sernbo, Olavi \\ Suomalainen, Agnar Tegnander, for The Hyaluronan Multicentre Trial Group ${ }^{\star}$
}

\begin{abstract}
Objective-To assess the effects of intraarticular injections of hyaluronan on symptoms of knee osteoarthritis (OA). Methods-Two hundred and forty patients with symptomatic, radiological knee OA were randomly assigned to treatment with weekly injections for five weeks with either $25 \mathrm{mg}$ of high molecular weight hyaluronan or vehicle. Results were evaluated at weeks $1,2,3,4,5,13$, and 20 by visual analogue scales (pain, function, motion, activity), algofunctional index, and global evaluation by patient and investigator. Analysis was by 'intention to treat', 'per protocol', and area under the curve principles on unstratified patient groups and for patients stratified into four groups of equal size by age and baseline algofunctional index.

Results-No serious side effects were reported. At 20 weeks both treatment groups were improved compared with baseline, with no difference between unstratified groups treated with placebo or hyaluronan. Comparison of treatment groups stratified by age and baseline algofunctional index revealed a significant difference in favour of hyaluronan over placebo (pain, activity, algofunctional index, global evaluations by patient and investigator) for patients older than 60 years and with a baseline algofunctional index greater than 10. There was no clinically relevant difference between the two treatments for the other three stratified subgroups of younger age or fewer symptoms. Similar results were obtained by area under the curve, intention to treat, and per protocol analysis.

Conclusions-Patients older than 60 years with knee osteoarthritis and with significant symptoms corresponding to an index of severity of knee disease of 10 or more, comprise the group most likely to benefit from treatment with intra-articular hyaluronan injections.
\end{abstract}

(Ann Rheum Dis 1996; 55: 424-431)
The total social costs of osteoarthritis in the United States have been estimated to approach $1 \%$ of the gross national product. ${ }^{1}$ Treatment of this condition includes walking aids, training, physical therapy, pharmacological agents, and surgery. Many of the commonly practised non-invasive, non-pharmacological methods lack adequate documentation of efficacy. ${ }^{2}$ Surgical methods of preserving and restoring articular cartilage are practised, but their effectiveness has only rarely been proven in randomised, controlled trials. ${ }^{3}$ Joint arthroplasty is an excellent treatment for advanced knee osteoarthritis, ${ }^{4}$ but is costly and not suitable for all patients. Analgesics and nonsteroidal anti-inflammatory drugs (NSAIDs) are used widely, but their efficacy has been questioned, ${ }^{5-8}$ and the frequent and serious adverse effects of NSAIDs in the elderly have recently been highlighted..$^{9-11}$

These problems have stimulated a search for alternative, symptom modifying drugs for use in osteoarthritis. Hyaluronan is a natural constituent of joint fluid and all connective tissues. Randomised, controlled clinical trials of intraarticular injections of hyaluronan for knee osteoarthritis have shown variable results, those from some trials suggesting long lasting pain relief, ${ }^{12-14}$ while others have failed to show a difference between hyaluronan and placebo. ${ }^{15} 16$ These trials have included patients of varying age and with varying disease severity, ranging from those with cartilage changes and knee pain, but no radiological signs of osteoarthritis, ${ }^{16}$ to patients with classical osteoarthritis. ${ }^{14}$

We have studied 240 patients with symptomatic, radiological osteoarthritis of the knee in a randomised, placebo controlled, double blind, multicentre clinical trial, and compared the effect of intra-articular injections of hyaluronan and placebo on knee symptoms and function.

\section{Patients and methods}

STUDY MEDICATION

Hyaluronan $25 \mathrm{mg}$ (approximate $M_{\mathrm{r}}$ about 1000 kDa) (Artzal ${ }^{\circledR}$ batch 0X555A, Astra Läkemedel, Södertälje, Sweden) was supplied as a sterile $1 \%$ solution in $2.5 \mathrm{ml}$ phosphate buffered saline, 
$\mathrm{pH}$ 7. Placebo consisted of $2 \cdot 5 \mathrm{ml}$ of the phosphate buffered saline vehicle, $\mathrm{pH} 7$.

\section{PATIENT SELECTION}

The study was performed in accordance with the Declaration of Helsinki and the Nordic guidelines Good Clinical Trial Practice. ${ }^{17}$ After approval by the local ethics committees and with informed patient consent, 240 patients (106 men, 134 women) were enrolled from eight orthopaedic or rheumatology clinics in Denmark, Finland, Norway, and Sweden. Patients at each centre were allocated randomly to two parallel groups of equal size, one of which received five weekly injections of the active substance, hyaluronan, and the other an equal number of injections of vehicle only. All patients had a clinical history of symptomatic, radiologically verified unilateral osteoarthritis of the knee (50-100\% obliteration of the medial tibiofemoral joint space without bony erosion on anteroposterior standing films at $10-15^{\circ}$ flexion, taken within six months of the start of the study). Each centre aimed to recruit at least 12 patients who were suitable for treatment efficacy evaluation. The hip-kneeankle angle at baseline was calculated for all patients from anteroposterior fully weight bearing radiographs, in order to obtain an assessment of the degree of angular deformity; it was measured as the lateral angle between a line drawn through the midpoint of the femoral head and the tibial spines, and a line drawn through the midpoint of the tibial spines and the talus. An angle of more than $180^{\circ}$ denotes a varus knee alignment. Minimum tibiofemoral joint space was measured by millimetre rule on anteroposterior fully weight bearing films taken at $10-15^{\circ}$ flexion.

Inclusion criteria were: age $40-75$ years at inclusion; symptomatic, radiologically verified knee osteoarthritis (stage I-II according to Ahlbäck ${ }^{18}$ ); knee pain on the day of examination scoring more than $10 \mathrm{~mm}$ on a $100 \mathrm{~mm}$ visual analogue scale (VAS) at baseline; and an algofunctional score of 4 or greater at baseline. ${ }^{19}$

Exclusion criteria were: significant symptoms of osteoarthritis of both knees; previous intra-articular fracture of the knee; rheumatoid arthritis or other inflammatory arthritis as diagnosed by American College of Rheumatology criteria, ${ }^{20}$ including $\mathrm{C}$ reactive protein and serum rheumatoid factor concentrations; intra-articular injections of steroids or any other invasive procedure in the knee within the previous six months; any other condition that might interfere with the efficacy assessment or completion of the trial.

\section{PATIENT STRATIFICATION}

After completion of the data collection, but before the treatment code was broken, several baseline variables of potential prognostic value were identified: age, gender, algofunctional index, centre. On the basis of the mean age of the whole patient group at inclusion and the mean level of symptoms and function at base-
Baseline characteristics for all 240 randomised patients

\begin{tabular}{|c|c|c|}
\hline Variable & Hyaluronan & Placebo \\
\hline (ears) & $58.53(8 \cdot 34)$ & $58.03(8.44)$ \\
\hline & 170 . & 169 \\
\hline ght $(\mathrm{kg})$ & $80 \cdot 58(12 \cdot 88)$ & $78 \cdot 51(14 \cdot 10)$ \\
\hline $1 / \mathbf{F}$ & $53 / 67$ & $53 / 67$ \\
\hline duration & $28.59(2 \cdot 88)$ & $28 \cdot 12(4 \cdot 40)$ \\
\hline & 64 & $60 / 60$ \\
\hline & 100 & 109 \\
\hline HK & $(4 \cdot 80)$ & $186 \cdot 03(4 \cdot 77)$ \\
\hline \multicolumn{3}{|l|}{ Joint space width (mm) } \\
\hline Lateral & & \\
\hline & & 26) \\
\hline Baseline joint $f$ & $5.68(11.43)$ & $4 \cdot 65(9 \cdot 05)$ \\
\hline \multicolumn{3}{|l|}{ Baseline VAS (mm } \\
\hline Pain & 44. & 42 \\
\hline Activi & 60 . & 60 \\
\hline Knee f & $51 \cdot 15(21$. & $49 \cdot 28(21$. \\
\hline Range of & $33.78(22.36)$ & $38.49(24.6$ \\
\hline Baseline Lequèsne i & & $9.56(3.63$ \\
\hline Clinical examinat & $20 \cdot 82(5 \cdot 30)$ & $21.02(5.51)$ \\
\hline & $54 \cdot 12$ & $57 \cdot 94(15 \cdot 74)$ \\
\hline & 1.5 & $2 \cdot 60(1.58)$ \\
\hline
\end{tabular}

Values are mean (SD) or number of patients.

HKA = Hip-knee-ankle; VAS = visual analogue scale

line, as assessed by the algofunctional index (table), ${ }^{19}$ a stratification was made, generating four groups of approximately equal size: patients aged 40-60 years with algofunctional index 4-10; those aged 40-60 years with algofunctional index greater than 10; patients aged 61-75 years with algofunctional index 4-10; those aged 61-75 years with algofunctional index greater than 10 (fig 1). The selection of these particular stratification criteria was based on the identification of significant interactions for these factors ${ }^{21}$ and their ease of use in everyday clinical practice. No significant interactions were found for gender or centre. Radiological data were not used as a basis for stratification because of the poor reproducibility in this setting and the limited relationship between radiological findings and severity of symptoms in the individual patient.

\section{STUDY DESIGN}

Routine blood and urine laboratory values were investigated at a visit about three weeks before the start of the study, and again one week after the last trial injection. After the study start, patients were seen weekly for five weeks (weeks 0-4), at one week after the last injection (week 5), and at 13 and 20 weeks after the first injection.

Stratification

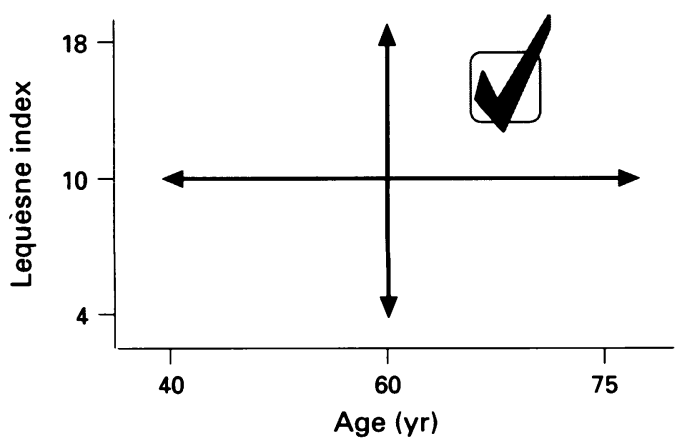

Figure 1 Stratification of patients by age and baseline Lequèsne index of severity of knee disease. ${ }^{19}$ Ticked quadrant represents patients aged more than 60 years and with a Lequèsne index of severity of knee disease greater than 10 at baseline. 
At weeks $0,1,2,3$, and 4 , the patients were given an intra-articular injection of $2.5 \mathrm{ml}$ of active drug or placebo. A small amount of local anaesthetic was used to anaesthetise the skin before the intra-articular injection, and any effusion was aspirated before the injection. The patient was blinded to the injection of drug or placebo. The injection was given by an investigator who took no part in the safety or efficacy assessments either before or after the injections. The examiner responsible for safety and efficacy assessments was not present during the treatment. In this way, neither the patient nor the examiner was aware of the nature of the treatment, and appropriate double blinding was asssured.

Concurrent and escape medication in the form of simple analgesics (for example paracetamol), in addition to non-steroidal antiinflammatory drugs, was allowed during the trial, and the identity and amount of drug taken was noted in the case record forms at each visit. No additional intra-articular injections in any of the knees were allowed during the study. Clinical assessments were performed immediately before aspiration of the joint and injection at weeks $0-4$, and at weeks 5,13 , and 20 after the first injection.

Primary efficacy variables were an algofunctional index, ${ }^{19}$ together with $100 \mathrm{~mm}$ VAS values for knee pain, range of motion, activity level, and total knee function on the day of examination as assessed and marked by the patient at each visit. The algofunctional index has been validated for knee osteoarthritis and is based on three parts that separately assess knee pain, walking distance, and activities of daily living function, each on a scale of $0-8$, so that 0 represents no pain and full function, and 24 represents maximal pain and disability. ${ }^{19}$

Secondary variables were: activity level and a knee function score examined at 0 and 20 weeks; ${ }^{22} 23$ a score based on a standardised clinical examination of the knee; quantity of concurrent medication; global assessment by patient and examiner; number of leucocytes in joint fluid, and volume of joint fluid aspirated.

A checklist and direct question technique to monitor adverse events and local reactions was used by an examiner blinded to the treatment. Any serious adverse events were reported to the principal investigator and the sponsor, and the possible need to withdraw the patient from the study was considered.

POWER CALCULATIONS, DATA HANDLING, STATISTICAL METHODS

The required number of patients was calculated on the basis of the expected variations of the VAS efficacy responses, and from the expected responses in the index of severity for osteoarthritis of the knee. ${ }^{19}$ It was assumed that the treatment difference would be $12 \mathrm{~mm}$ VAS pain (36 $\mathrm{mm}$ for the placebo group and $24 \mathrm{~mm}$ for the active group). The standard deviation was assumed to be $25.8 \mathrm{~mm}$ in both groups. With an $\alpha$ value of 0.05 and a $\beta$ value of $0 \cdot 10$ (power $=90 \%$ ), this would require 200 patients suitable for evaluation of treatment efficacy, randomised equally to the two treatment groups. The algofunctional index should have a smaller coefficient of variation than the VAS variable ( $40 \%$ compared with $60-70 \%$ ). We thought it clinically interesting to establish a narrower difference in mean response between placebo and active group (that is, $20 \%$ ), as the algofunctional index includes information regarding the functional status of the patient. ${ }^{19}$ Taking the same $\alpha$ and $\beta \mathrm{p}$ values, this would require 85 patients in each groupthat is, 170 patients. A total of 200 patients should therefore be a suitable number of patients under the assumptions given.

Participants from all centres met before the start of the study in order to standardise the injection technique and assessment procedures. All case record forms to be filled in by the patient were translated into the local language, while case record forms to be filled in by the investigators were in English. Verification of source data was made near the completion of the study. An extensive data validation programme was used to check for inconsistent data in the computer based data file.

Statistical evaluations were 'per protocol' (that is, considering all inclusion and exclusion criteria), and by the 'intention to treat' principle, whereby all patients included and randomised (except one lost to follow up) were used. In the per protocol evaluations, patients were included who had at least four injections (any experiencing early treatment failure resulting in termination of treatment before injection four because of lack of effect were excluded), baseline VAS for pain more than 10 $\mathrm{mm}$, baseline algofunctional index equal to or more than 4 , and no major protocol violations. All tests were two sided and $p$ values less than 0.05 were considered significant; between group differences and correlations that are described as significant were always significant at $p<0.05$. No adjustments for multiple comparisons were made. The method of carrying forward the last value for each variable was used to avoid having missing values at the different time points.

Non-parametric methods were used, as they do not rely on the assumptions of normality and linearity that tests such as analysis of variance and ANCOVA do. Wilcoxon two sample tests were thus used for all continuous or ordinal data. In order to adjust for possible differences at baseline (regardless of whether these were significant or not), the changes from baseline values were used for Lequèsne index and VAS values. $\chi^{2}$ tests (or Fisher's exact test when appropriate) were used for dichotomous data.

Tests were made for each time period (up to days $12,18,26,33,51,105$, and 185-corresponding to weeks $1,2,3,4,5,13$, and 20 ). These day limits were decided before code breaking and selected so that an absolute minimum of ambiguities existed. Because multiple tests at different time points are not ideal from a statistical point of view, we complemented these with an overall measure of treatment effect using an area under the curve 
(AUC) technique. This was used for Lequèsne algofunctional index and VAS scores. An interpolation between the observations for each patient up to the last visit before day 185 was made, and the mean change from baseline up to the last measurement was calculated. This constituted a theoretical mean decrease in the measurement variable experienced by the patient for the whole 20 week study period up to the last visit. The results of these calculations were compared between treatment groups using Wilcoxon two sample tests.

For analysis by the intention to treat principle, the last valid value up to day 185 was used for the change in Lequèsne index, global assessments by patient and investigator, and changes in VAS scores. This was also the case for the four subgroups generated by stratification for age (less than/greater than 60 years) and baseline Lequèsne index (less than/greater than 10).

\section{Results}

NUMBER OF PATIENTS, BASELINE DATA

A total of 240 patients from eight centres were included in the study. The number of patients enrolled at each centre varied between nine

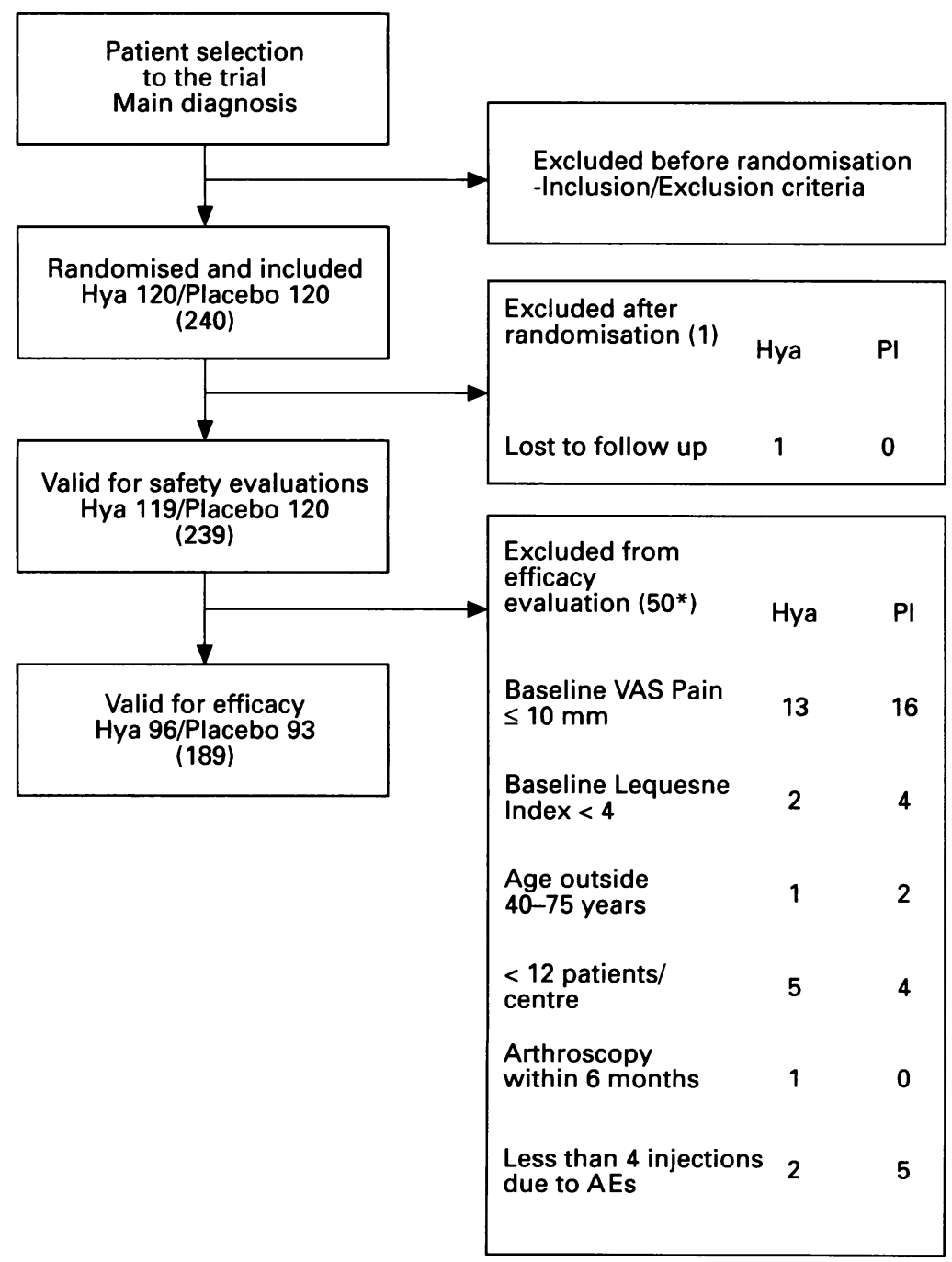

Figure 2 Flow chart for randomised patients, showing dropouts at different stages of the trial, and the reasons. (Patients can have several reasons for exclusion, therefore the number of patients in the table 'Excluded from efficacy evaluation' exceeds 50.) Hya = Hyaluronan treatment; $P l=$ placebo; $V A S=$ visual analogue scale; $A E=$ adverse event. and 61 . One hundred and eighty nine patients were suitable for evaluation of treatment efficacy at the conclusion of the study. Fifty one patients were not valid per protocol for efficacy (fig 2): eight of them (three receiving hyaluronan and five receiving placebo) prematurely discontinued treatment - that is, they did not receive five injections. Seven patients discontinued the trial because of adverse events, and one patient received one injection of hyaluronan, but did not return for follow up.

The table shows the patient characteristics at baseline for all patients randomised to the treatment groups. The treatment groups were comparable with respect to all variables, but there were minor but statistically significant differences for osteoarthritis stage ${ }^{18}$ and medial joint space width.

\section{TREATMENT EFFICACY FOR UNSTRATIFIED} GROUPS

At 20 weeks, both groups had improved compared with baseline by per protocol analysis of all patients suitable for efficacy evaluation (fig 3). There were no differences between the hyaluronan and placebo groups with regard to the measures of efficacy when no stratification was made. Analysis of data by intention to treat principles gave similar results. Because of the significant interactions observed for age and baseline algofunctional index, stratifications by age and baseline algofunctional index were made as described to yield four subgroups of approximately similar size.

TREATMENT EFFICACY FOR STRATIFIED GROUPS Differences between treatment groups at discrete evaluation time points are not reported, unless a significant difference was also found for the variable in question by the AUC technique of data analysis. No significant differences between treatments were found for VAS range of motion and VAS knee function.

Visual analogue scales for pain-The older age group (over 60 years) with a baseline Lequèsne algofunctional index of more than 10 , exhibited

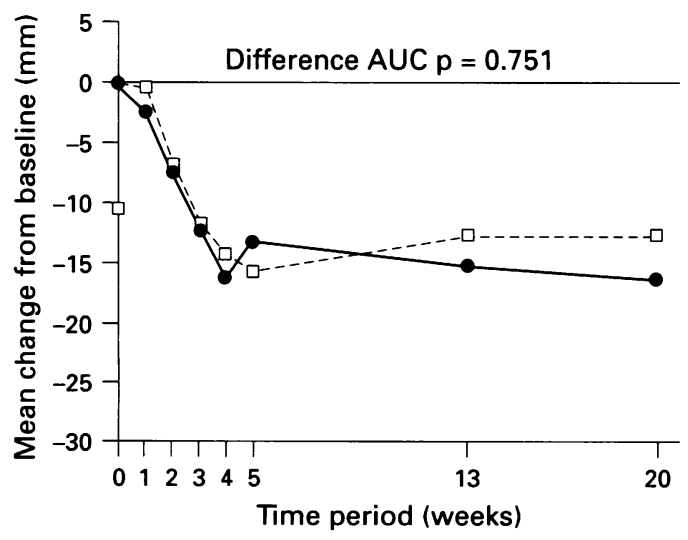

Figure 3 Mean change from baseline visual analogue scale data for pain in the hyaluronan (O) and placebo (口) treated groups (unstratified). Values for $p$ for differences between groups: week $1=0 \cdot 260$; week $2=0.941$; week $3=0.923$; week $4=0.840$; week $5=0.376$; week $13=0.608$; week $20=0.538$ 


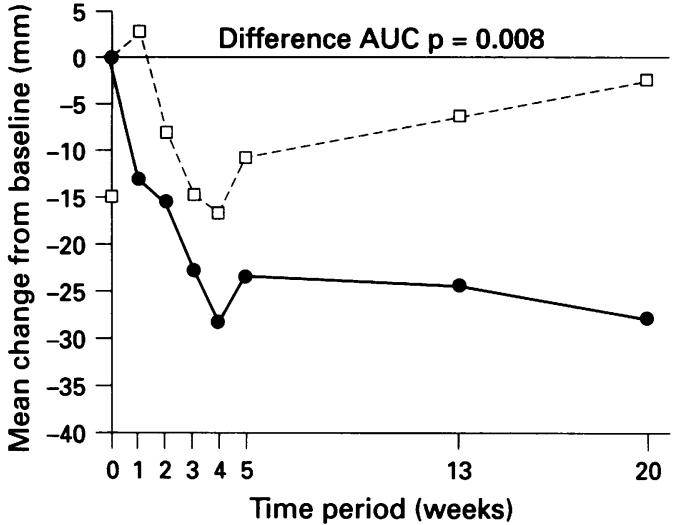

Figure 4 Mean change from baseline visual analogue scale data for pain in the hyaluronan (O) and placebo ( $\square$ ) treated stratified subgroups comprising patients 60-75 years old and with a baseline Lequèsne index greater than 10. Values for $p$ for differences between groups: week $1=0.008$; week $2=0.387$; week $3=0 \cdot 181$; week $4=0.090$; week $5=0.070$; week $13=0.014 ;$ week $20=0.004$

an advantage of hyaluronan over placebo for VAS pain at weeks $1 \quad(p=0.008), 13$ $(p=0.014)$, and $20(p=0.004)$ (fig 4). Analysis by AUC for mean treatment effect over 20 weeks similarly found an advantage for hyaluronan ( $p=0.008$ ) (fig 4). At weeks 4 and 20 the mean reductions in pain VAS were more than $28 \mathrm{~mm}$. An analysis by the intention to treat principle likewise showed an advantage for hyaluronan over placebo for pain $(p=0.008)$ for this subgroup. No differences were found between treatments for the other three subgroups whether analysed by per protocol or intention to treat principles.

Visual analogue scales for activity-The subgroup older than 60 years with a baseline Lequèsne index greater than 10 showed a significant superiority for hyaluronan over placebo at weeks $2(p=0.047), 4(p=0.001), 5$ $(p=0.037), 13(p=0.030)$, and $20(p=0.028)$ (fig 5). A mean change in VAS for activity of $37 \mathrm{~mm}$ occurred at week 4, signifying a remarkable increase in activity. Analysis by AUC technique confirmed the advantage of hyaluronan over placebo for the whole treatment period $(p=0 \cdot 008)$. The superiority of hyal-

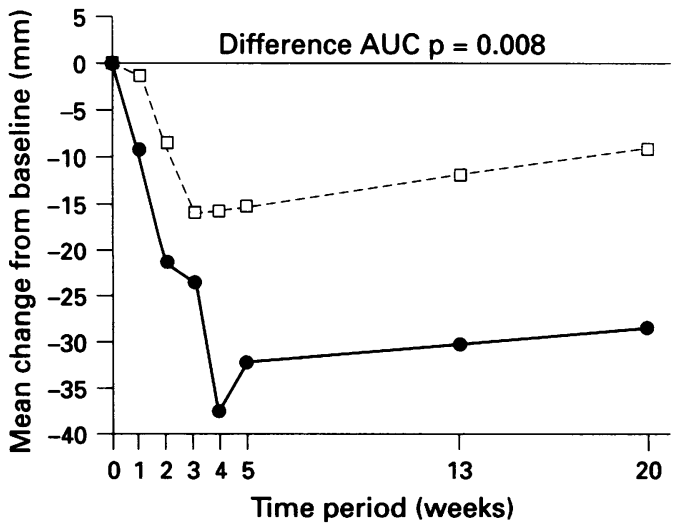

Figure 5 Mean change from baseline visual analogue scale data for activity in the hyaluronan () and placebo (口) treated stratified subgroups comprising patients 60-75 years old and with a baseline Lequèsne index greater than 10. Values for $p$ for differences between groups: week $1=0.117$; week $2=0.047$; week $3=0.232$; week $4=0.001$; week $5=0.037$; week $13=0.030$; week $20=0.028$.

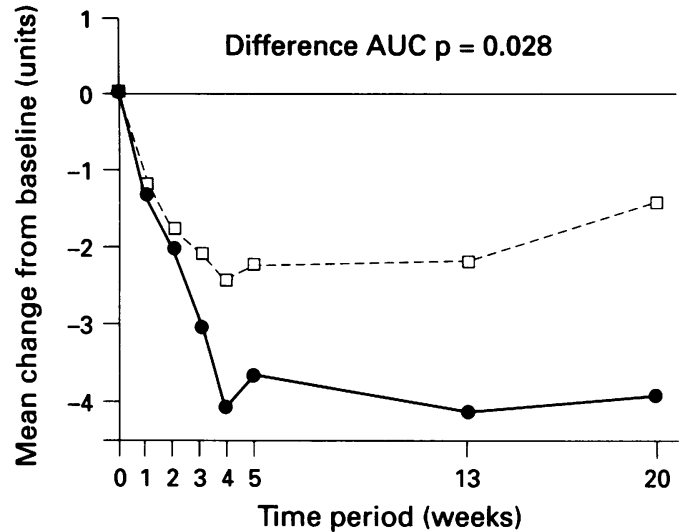

Figure 6 Mean change from baseline Lequèsne index of severity of knee disease in the hyaluronan (O) and placebo (D) treated stratified subgroups comprising patients 60-75 years old and with a baseline Lequesne index greater than

10. Values for $p$ for differences between groups: week $1=0.765$; week $2=0.317$; week $3=0.104$; week

$4=0.043$; week $5=0.165$; week $13=0.032$; week $20=0.056$.

uronan over placebo for this stratified subgroup was again confirmed by intention to treat analysis $(p=0.037)$. When analysed by AUC, the younger subgroup with a baseline Lequèsne index of 10 or less exhibited a difference in favour of placebo $(p=0.038)$. Placebo was also favoured at weeks $5(p=0.023)$ and $13(p=0.018)$ for this subgroup. There were no differences between treatments for the other subgroups.

Lequèsne index of severity of knee diseaseHyaluronan was superior to placebo at weeks $4(p=0.001)$ and $13(p=0.030)$ for the subgroup older than 60 years and with a baseline Lequèsne index greater than 10 (fig 6). Analysis of AUC revealed a mean reduction for the whole treatment period of 20 weeks in the hyaluronan treated patients of 3.5 units, compared with 1.9 units for placebo for this subgroup ( $p=0.028$ ) (fig 6). Analysis by intention to treat confirmed the advantage for hyaluronan over placebo $(p=0.025)$. There were no important differences between hyaluronan and placebo treatment for the other three subgroups, assessed at discrete time points or by AUC, or by per protocol or intention to treat.

A significant correlation coefficient of 0.68 (Pearson's) was found between pain assessed at 20 weeks by VAS and pain assessed by the pain section of the composite algofunctional index. ${ }^{19}$

Global assessments by patient and investigator-Stratification only by age yielded two subgroups in which the group older than 60 years showed an advantage for hyaluronan over placebo at the last visit (20 weeks) for global assessments (patient $p=0.019$, investigator $p=0.024)$. For the subgroup older than 60 years and with baseline Lequèsne index more than 10, hyaluronan showed a consistent pattern of superiority over placebo for both patient and investigator global assessments (data not shown), but statistical significance was observed only at week 20 (patient $p=0.037$, investigator $p=0.027$ ). Analysis by the intention to treat principle confirmed this advantage for hyaluronan $(p=0.042$ and 
$p=0.027$ for patient and investigator assessments, respectively). No differences between hyaluronan and placebo were found for the other subgroups.

LABORATORY EXAMINATIONS, ADVERSE EVENTS, CONCOMITANT MEDICATION

There were no differences of clinical importance between the two treatment groups with regard to blood and urine analysis.

No serious adverse events were reported, and no differences were found between the treatment groups in the frequency of other adverse events. However, there was a significant difference in favour of placebo when the maximum severity of injection site swelling was assessed $(p=0.041)$. Seven patients (five receiving placebo and two receiving hyaluronan) discontinued the treatment before their fifth injection because of adverse events.

No difference emerged with regard to consumption of analgesics or non-steroidal antiinflammatory drugs, for any reason, between the two treatment groups.

\section{Discussion}

Osteoarthritis is a heterogeneous condition, with a sometimes limited correlation between pathoanatomical features and the degree of symptoms. The specific sources and mechanisms of pain generation in the osteoarthritic joint are not clear and may, for example, be different in early and advanced stages of the disease.

Part of the conflict in the interpretation of the results of published trials of intra-articular injections of hyaluronan in knee osteoarthritis may arise from the inclusion of different osteoarthritic populations, or lack of adequate definition and description of the patient population included in the trial. Varying degrees of blinding and different methods of assessing outcome may add to the reported variation in efficacy. For example, while some trials have included patients with a combination of symptoms and radiological signs of knee osteoarthritis and reported the proportions of these groups, ${ }^{14} 15$ another trial focused only on younger patients with knee cartilage changes observed by arthroscopy in combination with symptoms suggestive of early stage osteoarthritis, but without radiographic signs of osteoarthritis. ${ }^{16}$ In yet other trials, the inclusion criteria and distribution with regard to osteoarthritis stage or the age of the patients have not been well described. ${ }^{12} 1324$ Other notable differences between published trials are the inclusion of patients with uni- or bilateral disease, and varying follow up times. All these differences complicate comparison and interpretation of the clinical trial data.

Yet another problem in trials involving intraarticular injections is the considerable placebo effect of injections and the resulting need to ensure blinding of both patient and examiner. ${ }^{25}$ In the present study, the patient was blinded to the type of injection given, and the investigator giving the injection did not take part in the examination of the patient before or after the injection. We therefore believe that our trial was appropriately masked with regard to both patient and examiner.

Only one previous trial has attempted to stratify the patients with regard to disease severity and outcome, ${ }^{15}$ and used radiographic stage of the knee osteoarthritis as the basis for stratification. Although no difference was found between hyaluronan and placebo in the unstratified patient groups, the results suggested a slower return to escape medication for those treated with hyaluronan than for those treated with placebo among patients with radiological changes corresponding to Kellgren and Lawrence ${ }^{26}$ stages I and II. No such difference was found for patients with more advanced radiological signs of osteoarthritis. The interpretation of these stratification data was, however, complicated by the considerable dropout rate $(38 \%)$ in this trial and the resulting small number of patients at follow up.

In the present trial, the rate of premature discontinuation of treatment was eight in 240, or $3 \%$. We have stratified for baseline age and Lequèsne index of severity of knee disease. These variables were chosen because of significant interaction, ${ }^{21}$ their ease of use in clinical work, and their potential relevance. Importantly, we found no interaction for gender or centre. The number of patients with a radiological stage II according to Ahlbäck ${ }^{18}$ was too small to form a basis for stratification. As there was no centralised reading of radiographs, we judged the precision of the medial tibiofemoral joint space measurements (in $\mathrm{mm}$ ) to be too poor to form a basis for stratification. Estimates of synovial fluid volume based on the amount withdrawn were also deemed too uncertain to be relied on for stratification purposes. ${ }^{27}$

We found no differences between the treatment groups in the unstratified data. However, the subgroup older than 60 years and with a baseline Lequèsne index of more than 10 , showed a consistent pattern of improvement in favour of hyaluronan over placebo. Significant advantages for hyaluronan were found with regard to VAS scores for pain and activity level, Lequèsne algofunctional index, and the global assessments by patient and examiner. These differences were reproduced both at discrete time points during follow up, and when assessed as mean treatment effect over the 20 week trial period by AUC measurements. Moreover, analysis by intention to treat principles of data for all randomised patients confirmed all the findings reached by analysis per protocol. No clinically relevant differences between treatments were found within the other stratified subgroups.

In a previous randomised, controlled, double blind clinical trial, we studied the effect of intra-articular hyaluronan injections in patients 30-64 years old with arthroscopically visible cartilage changes and chronic knee pain, but without radiological signs of osteoarthritis. ${ }^{16}$ The mean age of the patients in that trial was lower than that in the present trial (45 years and 58 years, respectively). Comparison 
of the patient data for the measurements of knee joint alignment (mean hip-knee-ankle angle $182^{\circ}$, compared with $186^{\circ}$ for the present trial) and medial tibiofemoral joint space (mean $4.5 \mathrm{~mm}$, compared with $2.3 \mathrm{~mm}$ for the present trial) confirmed the differences in the groups found by radiographic scoring according to Ahlbäck. ${ }^{18}$ The treatment protocol, drug, and blinding procedures were identical in the two trials and similar visual analogue scales for pain and activity were used in both trials We conclude from these two trials (of 52 and 20 weeks duration, respectively) that young patients with moderate symptoms and no or only early stage radiographic signs of osteoarthritis do not seem to derive any benefit from hyaluronan injections over that given by placebo injections only. This outcome contrasts with the significant benefit for hyaluronan over placebo that we found for older patients with definitive radiographic signs of osteoarthritis and significant symptoms. The results of both trials were confirmed by intention to treat analysis and the combined dropout rate in the two trials was 12 patients out of 292 , or $4 \%$. Although these results would appear to be statistically robust, their validity should be tested by trials focused on the responder group identified here.

For the stratified subgroup in which we found a significant advantage for hyaluronan over placebo, the mean improvement over the 20 week observation period was 3.5 units by the Lequèsne index. The mean improvement over 20 weeks in VAS pain was $23 \mathrm{~mm}$, and for VAS activity the mean improvement was $28 \mathrm{~mm}$. The mean maximum improvements for this subgroup were 4 units, $37 \mathrm{~mm}$, and $29 \mathrm{~mm}$, respectively. A mean improvement in Lequèsne index by 3.5 units over 20 weeks could, for example, be described as a complete loss of night pain combined with at least a doubled maximum distance walked. ${ }^{19}$ This might be regarded as a clinically relevant effect.

The reasons for the apparent difference in treatment efficacy by intra-articular injections in the stratified subgroups in this trial are unclear to us. However, the mechanisms of pain generation in the osteoarthritic joint are unknown, and might differ at different stages of disease development. In comparison, a short term trial of tiaprofenic acid and naproxen in osteoarthritis found responder rates of $52 \%$ and $59 \%$, respectively, with different responder characteristics for the two nonsteroidal anti-inflammatory drugs. ${ }^{28}$ Individual responder characteristics were also identified in an ' $n$ of 1 ' clinical trial comparing paracetamol and naproxen for osteoarthritis. ${ }^{8}$

Finally, we may only speculate on the mechanisms by which hyaluronan exerts its symptom modifying effects in this setting. We now know that many cells carry receptors for hyaluronan and that the molecule may affect cell-cell, cell-matrix and cell-receptor-ligand interactions. ${ }^{29}$ For example, interference with the hyaluronan receptor CD44 abrogated tissue oedema and leucocyte infiltration in a murine arthritis model. ${ }^{30}$ Other investigators have suggested that the viscoelastic properties of hyaluronan might act in lubrication at the joint surfaces. ${ }^{31}$ There is, as yet, little evidence to support any of these mechanisms as an explanation for the symptom relieving effects of intra-articular hyaluronan injections in osteoarthritic joints. Any hypothesis proposed would have to take into account the short intraarticular half life of the bulk of the injected hyaluronan,,$^{32}$ and the apparently long duration of the treatment effect.

^The Hyaluronan Multicentre Trial Group: Torbjörn Ahl, Nils Dalen (Department of Orthopaedics, Danderyd Hospital, 18288 Danderyd, Sweden); Christer Andersson, Magnus Lundberg, Magnus Odensten (Department of Orthopaedics, Linköping University Hospital, 58185 Linköping, Sweden); Linkors Lars Engebren, Agnar Tegnander (Department of Orthopaedics, Trondheim Regional Hospital, 7006 Trondheim, Norway); Gunna Englund, Kerstin Karlsson (Astra Läkemedel AB, 1518 Södertälje, Sweden); Martti Hämäläinen, Kalevi Koota (Department of Orthopaedics, Heinola Rheumatolog Foundation Hospital, 18120 Heinola, Finland); Erik Martin Jensen (Department of Rheumatology, Bispebjerg Hospital, 2400 Copenhagen, Denmark); Olof Johnell, Ingemar Sernbo (Department of Orthopaedics, Malmö University Hospital 21401 Malmö, Sweden); L Stefan Lohmander, Leif Ryd (Department of Orthopaedics, University Hospital, 22185 Lund, Sweden); Olavi Suomalainen, Urho Väätäinen (Department of Orthopaedics, Kuopio University Hospital, 70111 Kuopio, Finland).

Supported by the Medical Faculty of Lund Lniversity, the Swedish Medical Research Council, KaroBio AB, and Astra Läkemedel AB.

1 Yelin E. The economics of osteoarthritis. In: Brandt K D, Lohmander L S, Doherty M, eds. Osteoarthritis. Oxford: Lohmander L S, Doherty M, eds. Oste
Oxford University Press, 1996. In press.

2 Puett D W, Griffin M R. Published trials of nonmedical and noninvasive therapies for hip and knee osteoarthritis. Amn Intern Med 1994; 121: 133-40.

3 Buckwalter J A, Lohmander L S. Operative treatment of osteoarthrosis-current practise and future development. A current concept review. F Bone foint Surg Am 1994; 76 1405-18.

4 Knutson K, Lewold S, Robertsson O, Lidgren L. The Swedish knee arthroplasty register. A nation-wide study of 30,003 knees 1976-1992. Acta Orthop Scand 1994; 65: of 30,003

5 Bradley J D, Brandt K D, Katz B P. Comparison of an antiinflammatory dose of ibuprofen, an analgesic dose of ibuprofen, and acetaminophen in the treatment of osteoarthritis of the knee. N Engl f Med 1991; 325 $87-91$

6 Williams H J, Ward J R, Egger M J, et al. Comparison of naproxen and acetaminophen in a two-year study of treatment of osteoarthritis of the knee. Arthritis Rheum 1993; 36: 1196-206.

7 Dieppe P, Cushnagan J, Jasani M K, McCrae F, Watt I. A two-year, placebo-controlled trial of non-steroidal antiinflammatory therapy in osteoarthritis of the knee joint. inflammatory therapy in osteoarthrit

8 March L, Irwig L, Schwarz J, Simpson J, Chock C, Brooks P. n Of 1 trials comparing a non-steroidal antiinflammatory drug with paracetamol in osteoarthritis BMF 1994; 309: 1041-6.

9 Langman M J S, Weil J, Wainwright P, et al. Risks of bleeding peptic ulcer associated with individual nonsteroidal anti-inflammatory drugs. Lancet 1994; 343 1075-8

10 Rodriguez L A G, Jick H. Risk of upper gastrointestinal bleeding and perforation associated with individual nonsteroidal anti-inflammatory drugs. Lancet 1994; 343: 769-72.

11 Bateman D N, Kennedy J G. Non-steroidal antiinflammatory drugs and elderly patients. The medicine inflammatory drugs and elderly patients. The medicin

12 Dixon A S J, Jacoby R K, Berry H, Hamilton E B D. Clinical trial of intra-articular injection of sodium hyaluronate in patients with osteoarthritis of the knee. Curr Med Res Opin 1988; 11: 205-13

13 Dougados M, Nguyen M, Listrat V, Amor B. High molecular weight sodium hyaluronate (hyalectin) in osteoarthritis of the knee: a 1 year placebo-controlled trial. Osteoarthritis and Cartilage 1993; 1: 97-103.

14 Puhl W, Bernau A, Greiling H, et al. Intra-articular sodium hyaluronate in osteoarthritis of the knee: a multicenter double-blind study. Osteoarthritis and Cartilage 1993; 1: 243-52.

15 Henderson E B, Smith E C, Pegley F, Blake D R. Intraarticular injections of $750 \mathrm{kD}$ hyaluronan in the treatment of osteoarthritis: a randomised single centre double-blind of efficacy. Ann Rheum Dis 1994; 53: 529-34.

16 Dahlberg L, Lohmander L S, Ryd L. Intra-articular injections of hyaluronan in patients with early stage osteoarthritis of the knee. Arthritis Rheum 1994; 37: 521-8. 
17 Nordic Council on Medicines Publication 28. Good Clinical Trial Practice. Uppsala: Nordic Council on Medicines, 1989.

18 Ahlbäck S. Osteoarthrosis of the knee: a radiographic investigation. Acta Radiol Suppl 1968; 277: 7-72.

19 Lequèsne M G, Mery C, Samson M, Gerard $P$. Indexes of severity for osteoarthritis of the hip and knee. Validation-value in comparison with other assessment tests. Scand $\mathcal{f}$ Rheumatol 1987; (suppl) 65:

20 Arnett F C, Edworthy S M, Bloch D A, et al. The American Rheumatism Association 1987 revised criteria for the classification of rheumatoid arthritis. Arthritis Rheum 1988; 31: 315-24.

21 Fisher A C, Wallenstein S. Nonparametric tests for interaction and group differences in a two-way layout. $\mathcal{F}$ Biopharmaceut Statist 1991; 1: 17-25.

22 Tegner Y, Lysholm J, Odensten M, Gillquist J. Evaluation of cruciate ligament injuries. A review. Acta Orthop Scand 1988; 3: 336-41.

23 Lysholm J, Gillquist J. Evaluation of knee ligament surgery results with special emphasis on use of a scoring scale. Am f Sports Med 1982; 10: 150-4.

24 Grecomoro G, Martorana U, Di Marco C. Intra-articular treatment with sodium hyaluronate in gonarthrosis: a controlled clinical trial versus placebo. Pharmatherapeutica 1987; 2: 137-41.
25 Miller J H, White J, Norton T H. The value of intra-articular injections in osteoarthritis of the knee. $\mathcal{f}$ Bone foint Surg Br 1958; 40: 636-43.

26 Kellgren J H, Lawrence J S. Radiological assessment of osteo-arthrosis. Ann Rheum Dis 1957; 16: 494-502.

27 Geborek P, Saxne T, Heinegård D, Wollheim F A. Measurement of synovial fluid volume using albumin dilution upon intra-articular saline injection. $\mathcal{F}$ Rheumatol 1988; 15: 91-4.

28 Høyeraal $H$, Fagertun $H$, Ingemann-Hansen $T$, Ersmark H, Rönn O. Characterization of responders and nonresponders to tiaprofenic acid and naproxen in the treatment of patients with osteoarthritis. $\mathcal{F}$ Rheumatol 1993; 20: 1747-52.

29 Clark E A, Brugge J S. Integrins and signal transduction pathways: the road taken. Science 1995; 268: 233-9.

30 Mikecz K, Brennan F R, Kim J H, Glant T T. Anti-CD44 treatment abrogates tissue oedema and leukocyte infiltration in murine arthritis. Nature Medicine 1995; 1: 558-63.

31 Larsen N E, Lombard K M, Parent E G, Balazs E A. Effect of Hylan on cartilage and chondrocyte cultures. $\mathcal{f}$ Orthop of Hylan on cartilage

32 Fraser J R, Kimpton W G, Pierscionek B K, Cahill R N. The kinetics of hyaluronan in normal and acutely inflamed synovial joints: observations with experimental arthritis in sheep. Semin Arthritis Rheum 1993; 22: 9-17. 